\section{EL TREN ORBITAL: UN JUEGO EDUCATIVO BASADO EN UNA ANALOGÍA PARA APRENDER LA CONFIGURACIÓN ELECTRÓNICA EN SECUNDARIA}

\author{
The Orbital Train: An Educational Game Based On An Analogy to Learning the Electron \\ Configuration in Secondary Education
}

Revista eletrônica Ludus Scientiae - (RELuS) | V. 1, N. 2, Ago./Dez. 2017.

\section{AUTORES:}

ANTONIO JOAQUIN

FRANCO-MARISCAL ${ }^{1}$

ORCID 0000-0002-8704-6065

${ }^{1}$ Universidade de

Málaga

ROSARIO FRANCOMARISCAL ${ }^{2}$

Diferentes estudios muestran que la enseñanza-aprendizaje de la configuración electrónica se presenta como un tópico árido para el alumnado de educación secundaria. El uso de juegos educativos basados en analogías podría contribuir a superar algunas de las dificultades de aprendizaje en este tema. Este trabajo presenta la potencialidad del juego "El tren orbital" diseñado con este objetivo e implementado en el aula con una muestra de 41 estudiantes españoles de grado 10 (15-16 años). Los resultados ponen de manifiesto que la analogía en la que se basa el juego comparando las partes de un átomo con los elementos de un tren, ayuda parcialmente a subsanar algunos de los obstáculos en su aprendizaje al encontrar los alumnos elementos parecidos en su vida cotidiana con el que puede relacionarlos y entenderlos mejor. Asimismo, el juego es bien recibido en el aula, destacando los alumnos su gran utilidad e interés como sus principales virtudes.

Palabras clave: juego educativo, analogía, configuración electrónica, educación secundaria

\begin{abstract}
Different studies have shown that the teaching-learning of electron configuration is presented as an arid topic for secondary school students. The use of educational games based on analogies could help overcome some of the learning difficulties in this topic. This paper presents the potential of "The orbital train" game designed with this aim and implemented in the classroom with a sample of 41 Spanish students of grade 10 (15-16 years old). The results show that the analogy on which the game is based, by comparing the parts of an atom with the elements of a train, helps partially to overcome some of the obstacles in the learning because similar elements are found by students in their daily life to relate them and understand them better. Also, the game is well received in the classroom, highlighting the students its great usefulness and interest as its main virtues.
\end{abstract}

Key - Words: educational game, analogy, electron configuration, secondary education 


\section{INTRODUCCIÓN}

Diferentes estudios muestran que el estudio del átomo en los niveles básicos de la enseñanza de la química constituye uno de los temas más difíciles y complejos (Lee, 1993; GarcíaCarmona, 2006), siendo varias las razones que lo justifican. Por una parte, se debe a que los conceptos que se manejan no se encuentran en el ámbito macroscópico y cotidiano de los estudiantes (Rosado y García-Carmona, 2004) y por otra parte, a la capacidad de abstracción que se requiere para su aprendizaje, muy poco desarrollada en la etapa de educación secundaria (Pozo y Gómez-Crespo, 1998).

A pesar de ello, un contenido que resulta esencial en el estudio del átomo en la etapa de secundaria es la enseñanza/aprendizaje de la configuración electrónica de los átomos, ya que permite a los estudiantes adquirir un conocimiento básico y adecuado de la materia, debido a que las propiedades físicas y químicas de un elemento químico vienen determinadas por la distribución de los electrones en el interior del átomo (Eisberg y Resnick, 1994). A pesar de su interés didáctico, aún se consideran muy escasas las investigaciones educativas que abordan este tema (García-Carmona, 2006).

Una forma motivadora de introducir la estructura electrónica en el aula es a través de juegos educativos. Cabe destacar, el papel cada vez más destacado que están adquiriendo las investigaciones con juegos educativos en el ámbito de la didáctica de la química en los últimos años debido a su potencial didáctico (Antunes, Pacheco y Giovanela, 2012; Franco-Mariscal, 2016). Un tipo de juego interesante para abordar conceptos complejos y de naturaleza abstracta en el aula son los juegos educativos basados en analogías (Oliva, 2010). En este contexto lúdico, las analogías sirven como recurso para comprender una determinada noción o fenómeno, a través de las relaciones que establece con un sistema «análogo» que resulta para el estudiante más conocido y familiar (Dagher, 1995).

Una ventaja del empleo de juegos centrados en analogías en la enseñanza de los conceptos básicos de la teoría atómica moderna es que no requieren del uso de ideas matemáticas complejas como funciones de onda, números cuánticos u orbitales, que es una de las principales dificultades a las que se enfrenta el profesorado en su enseñanza. Conscientes de este problema, en la década de los 8o, Morwiek (1979) y Cervellati y Perugini (1981) estudiaron qué conocen o qué deberían saber los estudiantes de instituto sobre este tema. Según Morwiek (1979), los alumnos de secundaria deberían aprender la forma que tiene la región o espacio que ocupan los electrones en los distintos niveles de energía, así como ser capaces de describir varios niveles de energía (1s, 2s, 2p, etc.) Asimismo, los alumnos deberían pensar el enlace químico en término de solapamiento de orbitales, evitando el uso de argumentos matemáticos relacionados con funciones de onda y números cuánticos.

A pesar de que en la literatura se encuentran excelentes materiales, bancos de ideas o discusiones no matemáticas para trabajar en el aula de secundaria la teoría atómica moderna (Samoshin, 1998; Hanson, 2003; Trindade y Fiolhais, 2003), son escasos los trabajos basados en juegos educativos y analogías para abordar este tópico. Es por ello, que este trabajo pretenda exponer cómo las analogías aplicadas a juegos educativos pueden ser de utilidad para construir conocimientos en un dominio específico de la química. Más concretamente, se trata de mostrar cómo los elementos de un tren pueden servir de base para construir analogías que faciliten la comprensión de la configuración electrónica de los átomos.

\section{EL JUEGO EDUCATIVO}

El presente juego educativo tiene como objetivo didáctico la enseñanza-aprendizaje de la configuración electrónica de los átomos y está orientado para estudiantes de secundaria de 1516 años (grado 10). El juego utiliza como analogía un tren que realiza trayectos alrededor de una 
ciudad y consta de un tablero de mesa, 6o granos de arroz que se utilizan a modo de fichas y un dado.

El objetivo del juego es, dado un número determinado de pasajeros, averiguar qué vagones, compartimentos y asientos del tren están ocupados, en qué orden se han sentado los viajeros y en qué asiento se ha sentado el último pasajero.

El juego se presenta a los alumnos con esta historia: En una ciudad han inaugurado el llamado "Tren orbital", un peculiar medio de transporte que realiza trayectos por la periferia de la ciudad, pero que nunca llega a entrar a ella. Es peculiar debido a que los viajeros que se monten en él tienen que seguir al pie de la letra una serie de instrucciones, y en caso de no cumplirlas, el revisor les puede invitar educadamente a apearse en la parada más próxima.

\section{Descripción del tablero de mesa}

En primer lugar, se presenta a los estudiantes el tablero de mesa (figura 1) que representa el esquema del tren. El profesor pide al alumnado que lo describan e identifiquen todos los elementos que se pueden observar en él. La figura 2 muestra esta identificación realizada por un estudiante de grado 10.

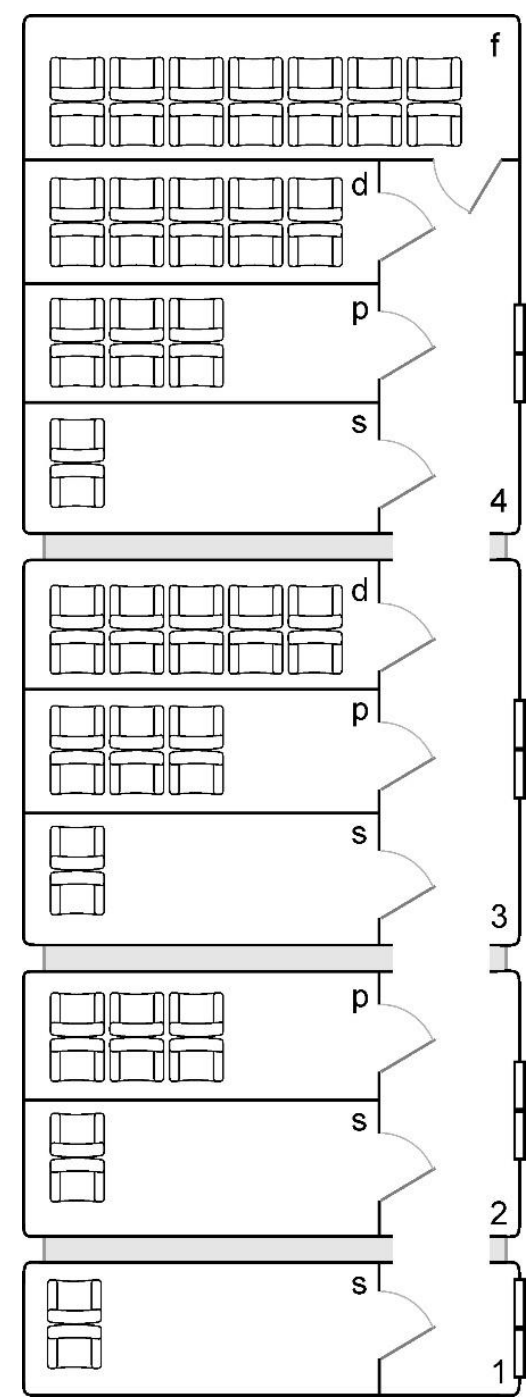

Figura 1: Tablero de mesa del juego "El tren orbital". Fuente: Elaborado por los autores. 


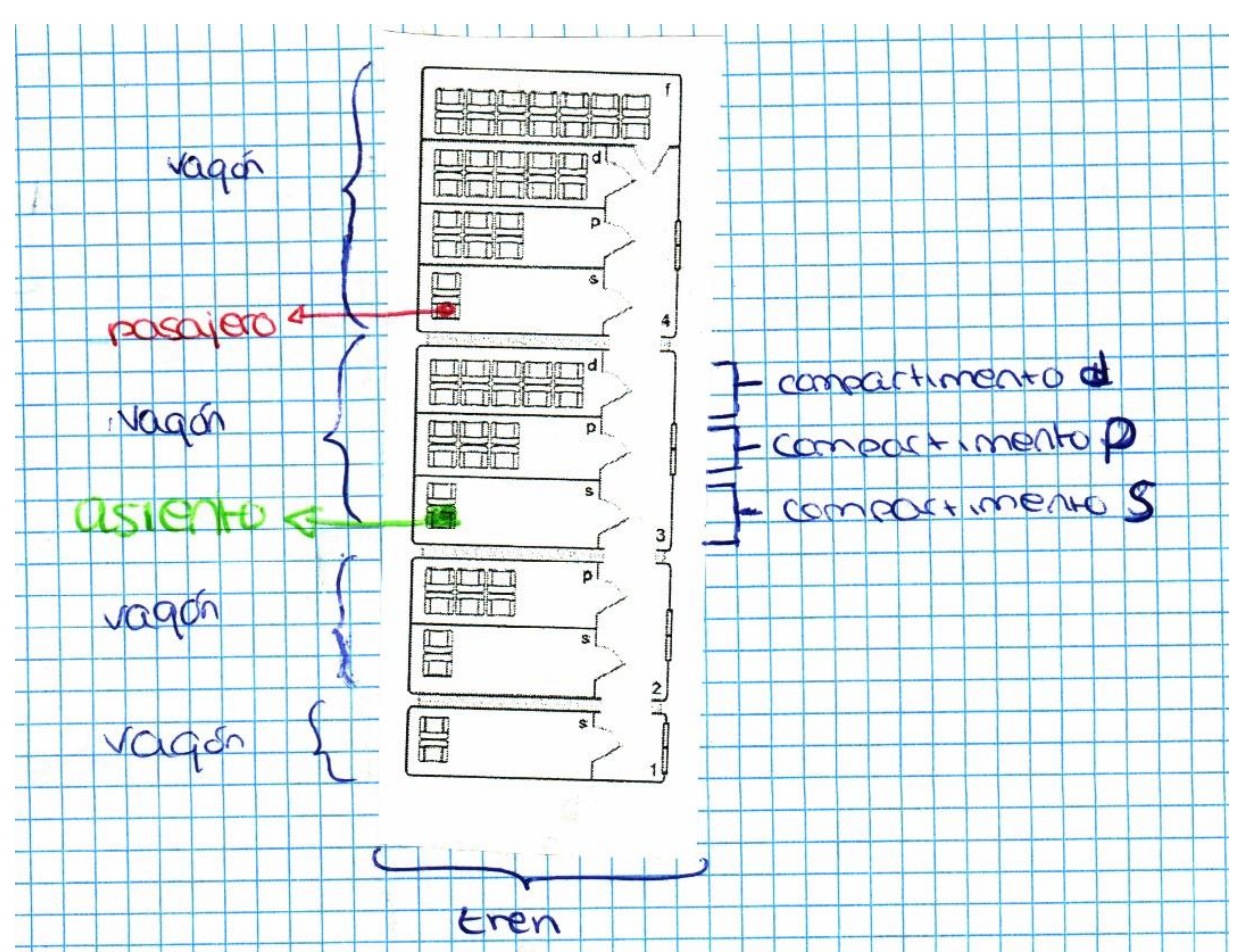

Figura 2: Identificación de los elementos del tren por un estudiante de grado 10. Fuente: Elaborado por los autores.

Se pretende con ello que los estudiantes interioricen los elementos del tren que conformarán posteriormente la analogía en la que se basa el juego. Concretamente, el alumnado debe indicar que el tren consta de cuatro vagones, cada uno de ellos con diferente número de asientos (2, 8, 18 y 32, respectivamente) y distinta distribución. Los asientos se disponen en cada vagón en varios compartimentos denominados $\mathrm{s}$ (sharp), $\mathrm{p}$ (principal), $\mathrm{d}$ (diffuse) y $\mathrm{f}$ (fundamental). Cada compartimento tiene un número determinado de asientos (s, dos; p, seis; d, diez y f, catorce).

El primer vagón tiene un único compartimento (s) con dos asientos (un par de asientos enfrentados entre sí). El segundo tiene dos compartimentos (s y p): uno con dos asientos con la misma disposición anterior y el otro con seis asientos (tres pares de asientos, es decir, tres mirando en la dirección del trayecto y tres mirando en sentido contrario). El tercer vagón tiene tres compartimentos (s, p y d) de dos, seis y diez asientos respectivamente. En este último compartimento existen cinco asientos en una dirección y otros cinco en la contraria constituyendo cinco pares de asientos en total. El cuarto vagón es el más amplio ya que cuenta con cuatro compartimentos (s, p, d y f) de dos, seis, diez y catorce asientos respectivamente, con la misma disposición anterior, además de siete asientos enfrentados a otros siete (siete pares de asientos en total). Los 10 y 14 asientos de este vagón están reservados para grandes personalidades, así que no van a ser ocupados habitualmente.

\section{Reglas del juego}

El número de jugadores en cada partida es entre 3 y 5 , actuando uno de ellos con el papel de revisor, que debe velar porque se cumplan correctamente las reglas del juego. Las instrucciones que deben seguir los viajeros al sentarse en su asiento son las siguientes:

1. Está prohibido que los viajeros permanezcan de pie durante el trayecto. 
2. Los viajeros pueden sentarse mirando en la dirección del trayecto o en sentido contrario. Si ambos están libres, debe ocupar el asiento en la dirección del trayecto.

3. Los viajeros se deben sentar comenzando desde el primer vagón, teniendo en cuenta que no se puede pasar al siguiente compartimento si no está lleno el anterior.

4. En los compartimentos en los que hay más de dos asientos, se irán colocando los viajeros lo más separadamente posible, respetando siempre las normas anteriores.

5. Como en los compartimentos los asientos se distribuyen por pares, se dice que si están ocupados los dos, hay una pareja de viajeros en ellos. En caso de que esté sentado un solo viajero, se dice que se trata de un viajero solitario.

6. Hay una excepción en el orden de ocupación de los compartimentos. El primer compartimento del cuarto vagón, se ocupa antes que el último compartimento del tercer vagón.

7. Los viajeros se deben bajar en orden inverso al de subida al tren, a excepción que bajarán antes los viajeros del primer compartimento del cuarto vagón seguidos de los del último compartimento del tercer vagón.

Conocidas estas reglas generales, el primer jugador lanza el dado y sube al tren tantos pasajeros como número haya salido en su tirada. Para ello, debe colocar a cada uno de los viajeros atendiendo a las normas indicadas. Una vez que los ha colocado, el revisor comprobará que todo está correcto. Si es así, le preguntará que indique la información de la tabla 1.

Tabla 1. Preguntas que hace el revisor a cada pasajero.

A. Distribución de viajeros por vagones.

B. Distribución de viajeros por compartimentos utilizando el número y letra de cada uno de ellos.

C. Posición exacta del último pasajero.

D. Número de pasajeros que quedan sentados por parejas y los que hay solitarios en el último compartimento ocupado.

E. Número de viajeros necesarios para completar las plazas disponibles del último compartimento con pasajeros.

Por ejemplo, imaginemos que el primer jugador saca un 6. Entonces deberá colocar sus viajeros de la forma mostrada en la figura 3: dos viajeros en el vagón 1 (compartimento s), otros dos en el vagón 2 (compartimento s) y dos más en el vagón 2 (compartimento p).

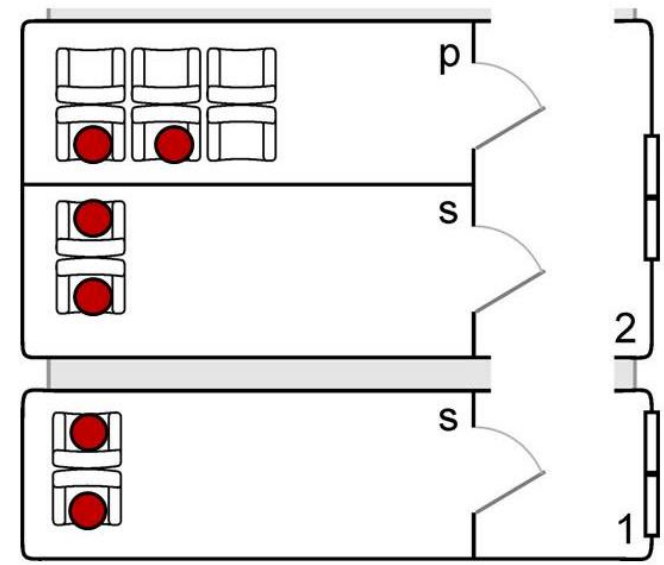

Figura 3: Ejemplo de una partida. Fuente: Elaborado por los autores. 
Como es correcto, el estudiante que actúa como revisor pedirá al compañero que indique la distribución de viajeros por vagones (2-4), su distribución por compartimentos $\left(1 \mathrm{~s}^{2} 2 \mathrm{~s}^{2} 2 \mathrm{p}^{2}\right)$, la posición exacta del último viajero $\left(2 \mathrm{p}^{2}\right)$, el número de pasajeros sentados por parejas (o) y solitarios en el último compartimento ocupado (2), y el número de viajeros necesarios para ocupar por completo el último compartimento con pasajeros (4). Si el estudiante responde correctamente a las cinco cuestiones obtendrá 1o puntos (2 puntos por pregunta). Si no es así, el revisor conseguirá tantos puntos como preguntas haya fallado.

A continuación tira el dado el segundo jugador que opera de la misma forma. A partir de la segunda ronda, el revisor preguntará al jugador después de lanzar el dado si quiere subir o bajar pasajeros, con idea de que pueda alcanzar la estabilidad de pasajeros en su vagón o compartimento. El jugador obtendrá una puntuación adicional de 1o puntos si en su jugada logra completar algún vagón. La partida concluye cuando se llega al asiento $4 \mathrm{p}^{6}$, momento en el que el jugador que haya obtenido la mayor puntuación resultará el ganador de la partida. El tiempo medio de una partida es de 15 minutos.

\section{Interiorización de las reglas}

Antes de empezar la primera partida, y con idea de interiorizar las reglas, que para algunos alumnos pudieran resultar complejas, se solicita a cada estudiante que realice la siguiente tarea en su cuaderno: "Ubica en los esquemas del tren que te facilitará tu profesor a los siguientes pasajeros: a) 8; b) 17 ; c) 34 . Indica en qué asiento se sienta cada uno de ellos y responde en todos los casos a las cinco cuestiones que le hará el revisor (ver tabla 1)”.

La figura 4 muestra el portafolio de uno de los estudiantes de grado 10 para el caso de 17 viajeros.

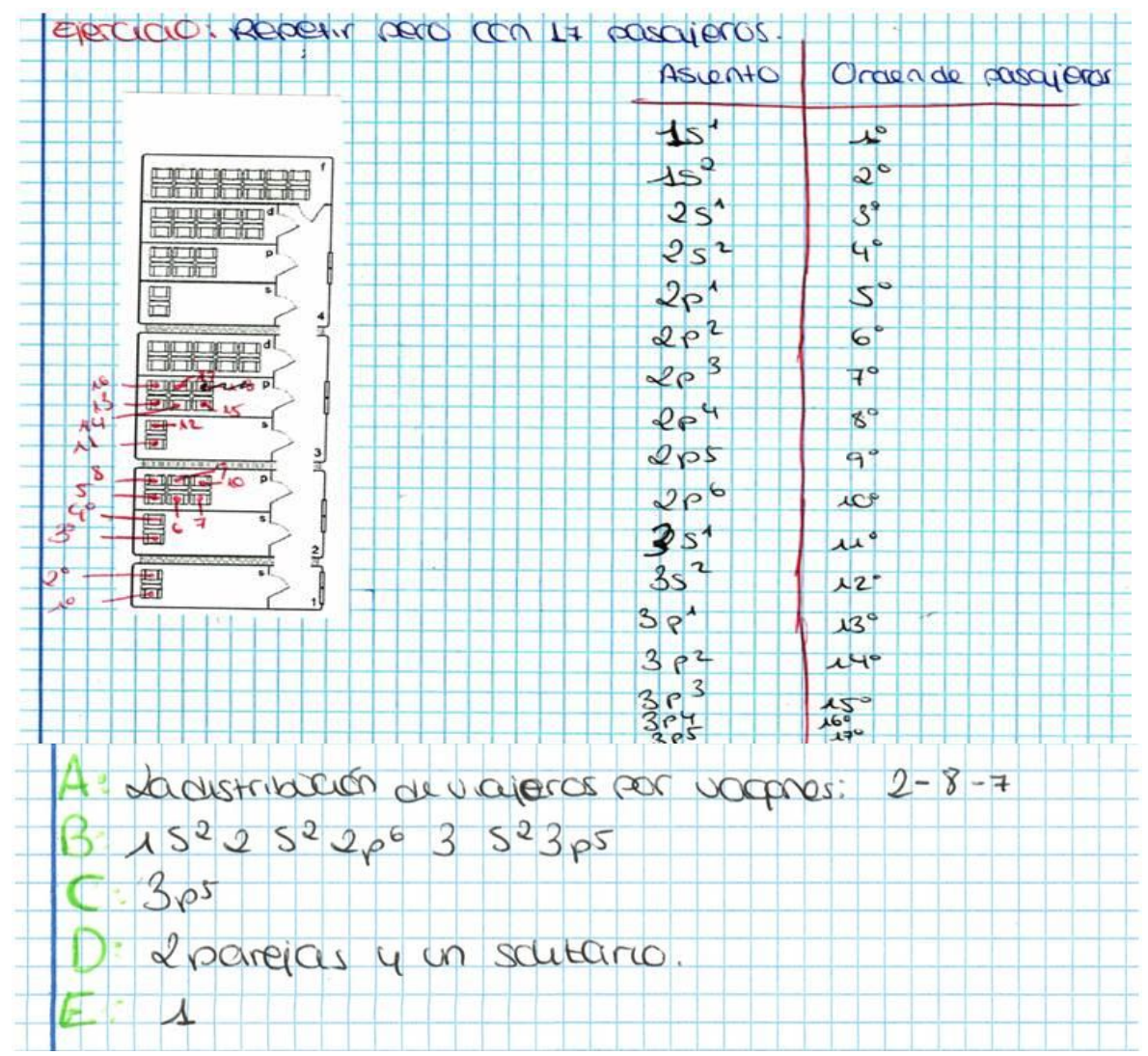

Figura 4: Portafolios de un estudiante de grado 10 para la tarea de 17 pasajeros. Fuente: Elaborado por los autores. 


\section{LA ANALOGÍA ENTRE LA CONFIGURACIÓN ELECTRÓNICA Y EL TREN}

El significado de la configuración electrónica de un átomo se puede entender más fácilmente si se compara con un tren. El símil empleado adopta una forma meramente visual entre lo que vemos en el mundo macroscópico (el tren) y lo que no se puede ver a nivel submicroscópico (el átomo) (figura 5).
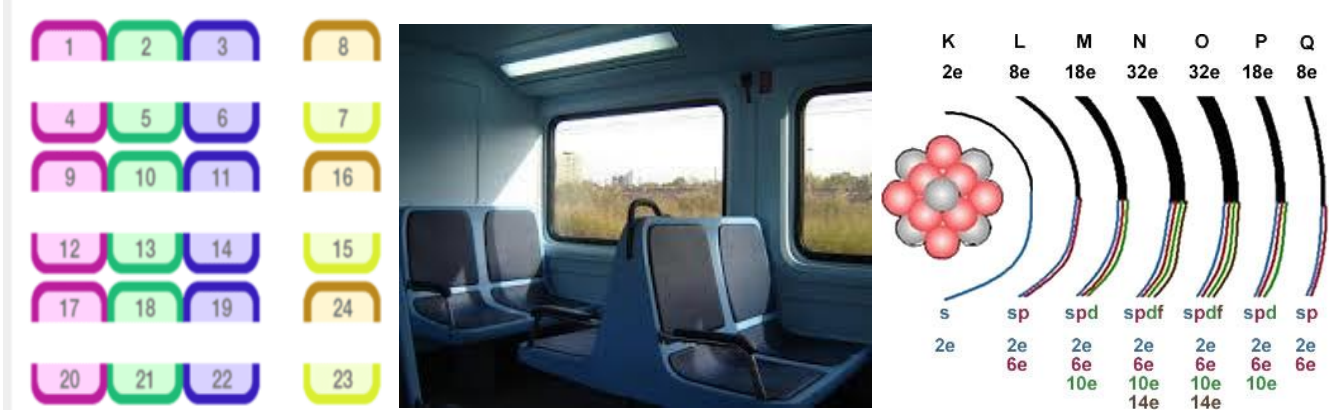

Figura 5: Formato gráfico mediante el cual surgió el símil propuesto en el juego. Fuente: Elaborado por los autores.

Como se observa en la figura 5, la distribución de los asientos de un tren (figura de la izquierda) y la configuración electrónica (figura de la derecha) comparten una similitud estructural (números, filas, secciones, etc.) que permite establecer una relación entre los dos sistemas. Las similitudes entre el tren y la configuración electrónica se recogen en la figura 6 y se detallan seguidamente.
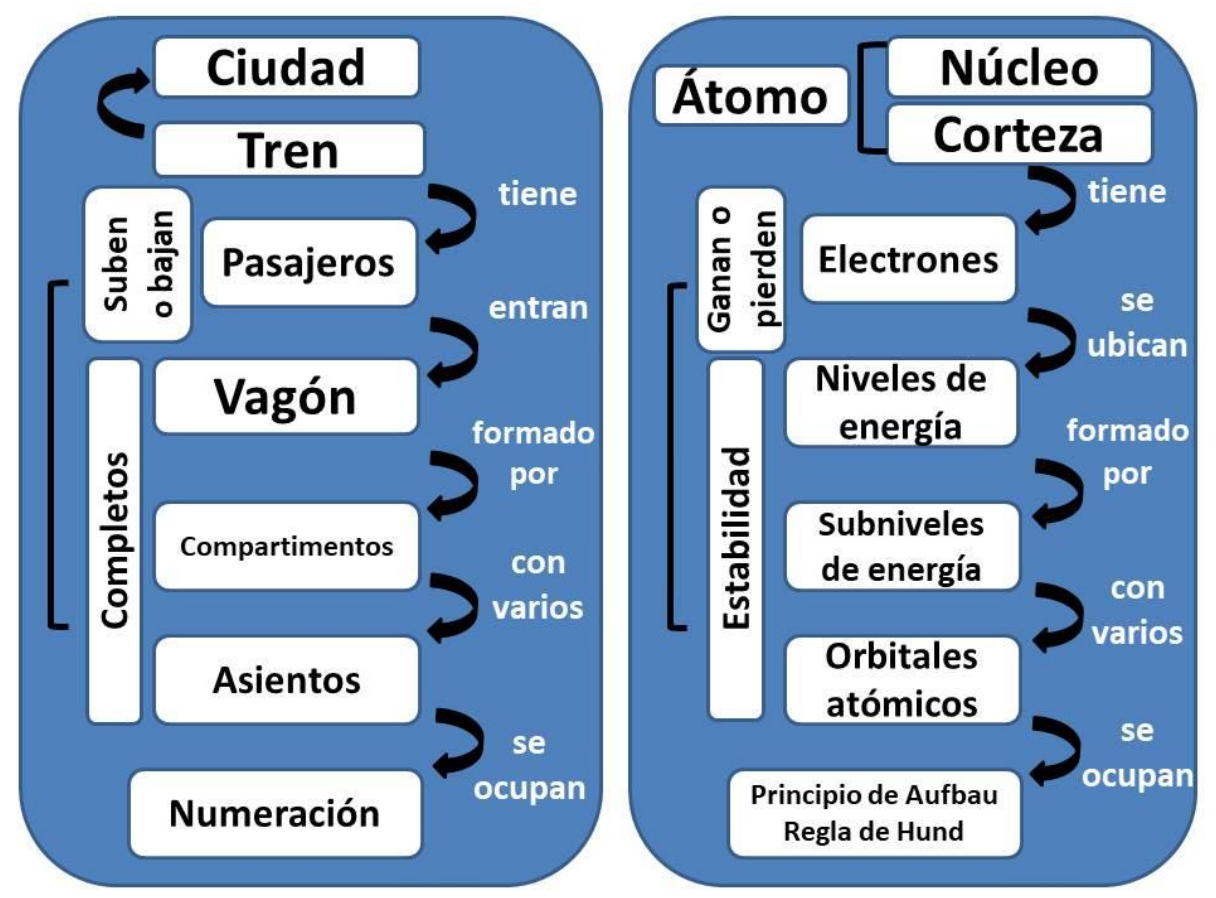

Figura 6: Relaciones implicadas en la analogía entre el tren y la configuración electrónica. Fuente: Elaborado por los autores. 
1. La ciudad y el tren se corresponde con un átomo neutro de un elemento.

2. La ciudad alrededor de la cual viaja el tren se asocia con su núcleo.

3. El tren se corresponde con la corteza del átomo.

4. Cada pasajero se asocia con un electrón.

5. La subida o bajada de pasajeros al tren corresponde con la ganancia o pérdida de electrones del átomo.

6. El número del vagón en el que entra un pasajero corresponde con un nivel de energía. Por ejemplo, el vagón número 2 equivale al nivel 2 de energía.

7. Cada vagón tiene varios compartimentos. Su número es variable en cada uno de los vagones. Cada compartimento se relaciona con un subnivel de energía (s, p, d ó f). Por ejemplo, dentro del vagón 2 (nivel 2) existen dos compartimentos. Se trata de los subniveles 2 y y 2p.

8. Cada compartimento tiene varios asientos. Cada par de asientos se corresponde con un orbital atómico.

9. Si todos los asientos de un compartimento están ocupados equivaldría a una situación de estabilidad atómica.

10. Las instrucciones que siguen los viajeros para sentarse se corresponden con las del Principio de Aufbau, que indica la ubicación de los electrones en los orbitales de un átomo, según el esquema de la figura 7 .

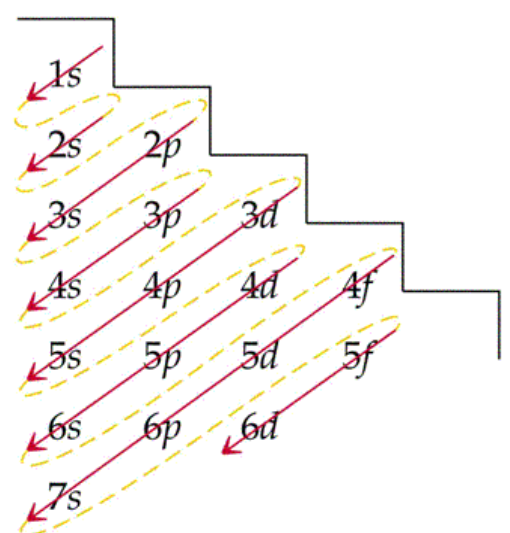

Figura 7: Principio de Aufbau. Fuente: Elaborado por los autores.

Además, los pasajeros se sientan atendiendo a la regla de Hund, según la cual ningún orbital puede tener dos electrones antes que los restantes orbitales de la misma subcapa tengan al menos uno. Además, se comienza con el orbital de nivel energético más bajo.

\section{DISEÑO DE LA INVESTIGACIÓN}

\section{Hipótesis de la investigación}

Esta investigación está focalizada en la hipótesis de que la analogía en que se basa el juego es eficaz para el estudio de la configuración electrónica en secundaria.

\section{Participantes}

El recurso planteado se evaluó como estudio piloto con una muestra de 41 estudiantes de grado 10 (15-16 años) que cursaban la materia optativa de Química en dos institutos de secundaria públicos de nivel socioeconómico medio de las provincias de Málaga y Cádiz (España). El 61\% eran chicos y el 39\% chicas. Todos ellos mostraban un perfil de estudiante trabajador y pretendían estudiar bachillerato de ciencias. Los estudiantes pertenecían a tres 
grupos de 10, 15 y 16 sujetos respectivamente, y se eligieron porque habían cursado en grado 9 los conocimientos básicos de la estructura de la materia y del átomo, excepto los relativos a la configuración electrónica.

El juego educativo fue implementado en el aula durante el curso 2017-2018 dentro de la unidad didáctica "El átomo" por los tres autores de este trabajo que actuaron como profesores observadores e investigadores. La figura 8 muestra algunas imágenes del desarrollo del juego en el aula.
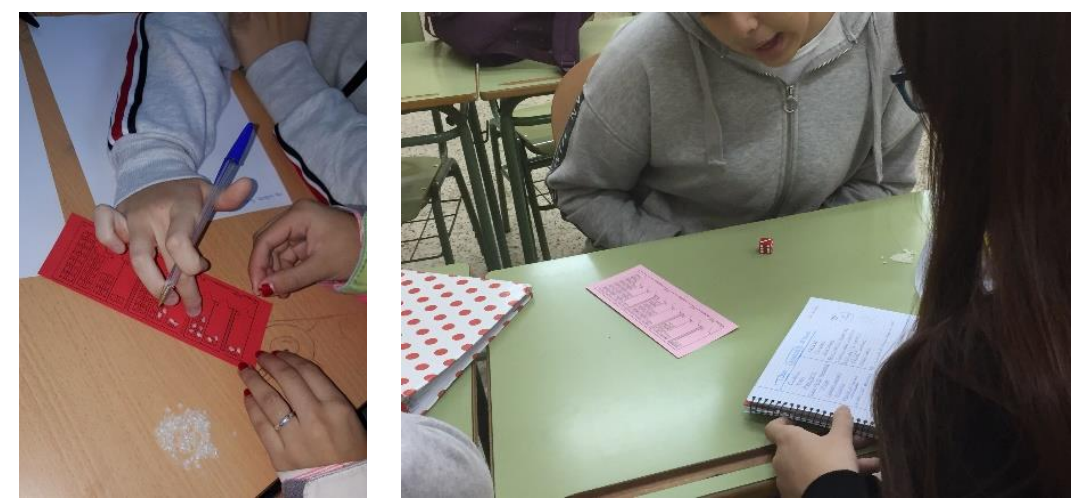

Figura 8: Estudiantes jugando en el aula. Fuente: Elaborado por los autores.

\section{Instrumentos}

Se realizó una evaluación de la analogía y del juego como recurso didáctico. La técnica de recogida de información en ambos casos fue el cuestionario. La tabla 2 recoge la cuestión que se planteó a los estudiantes tras la realización del juego sobre las similitudes entre ambos sistemas y las posibles correspondencias entre ellos, mientras que la tabla 3 muestra las cuestiones que se administraron a los alumnos para evaluar la potencialidad didáctica del juego.

Tabla 2: Cuestión planteada para evaluar la analogia.

Para entender mejor el átomo vamos a realizar una comparación entre el tren y el átomo fijándonos en las semejanzas. Para ello, une con flechas los conceptos de la columna de la izquierda con los de la columna de la derecha.

\begin{aligned} & \multicolumn{1}{c}{ Átomo } Juego educativo \\ & \hline Núcleo del átomo Pasajeros \\ & Corteza del átomo Numeración del asiento \\ & Electrones Tren \\ & Ganar y perder electrones Asientos \\ & Niveles de energía Compartimentos \\ & Subniveles de energía Vagón \\ & Orbitales atómicos Compartimentos completos \\ & Estabilidad Ciudad \\ & Principio de Aufbau y regla de Hund Subir y bajar pasajeros \\ & \hline\end{aligned}

Fuente: Elaborado por los autores. 
Tabla 3: Cuestionario de valoración del juego educativo.

- He aprendido...

- Lo mejor del juego

- Lo peor del juego

- Puntúa el juego de o a 10.

- Para cada una de las cualidades que se indica, valora el juego realizado:

$\begin{array}{lllll}\text { Sencillez: } & \circ \text { Muy poco } & \circ \text { Poco } & \circ \text { Algo } & \circ \text { Mucho } \\ \text { Utilidad: } & \circ \text { Muy poco } & \circ \text { Poco } & \circ \text { Algo } & \circ \text { Mucho } \\ \text { Atractivo: } & \circ \text { Muy poco } & \circ \text { Poco } & \circ \text { Algo } & \circ \text { Mucho } \\ \text { Interés: } & \circ \text { Muy poco } & \circ \text { Poco } & \circ \text { Algo } & \circ \text { Mucho }\end{array}$

Fuente: Elaborado por los autores.

\section{RESULTADOS}

\section{Evaluación de la analogia}

La figura 9 recoge en porcentajes de estudiantes, el número total de elementos de la analogía que son acertados por el alumnado. Como se aprecia, la curva obtenida tiene forma de campana de Gauss centrada en 4 elementos (con un 36.6\% de estudiantes), prácticamente la mitad de los elementos que componen la analogía.

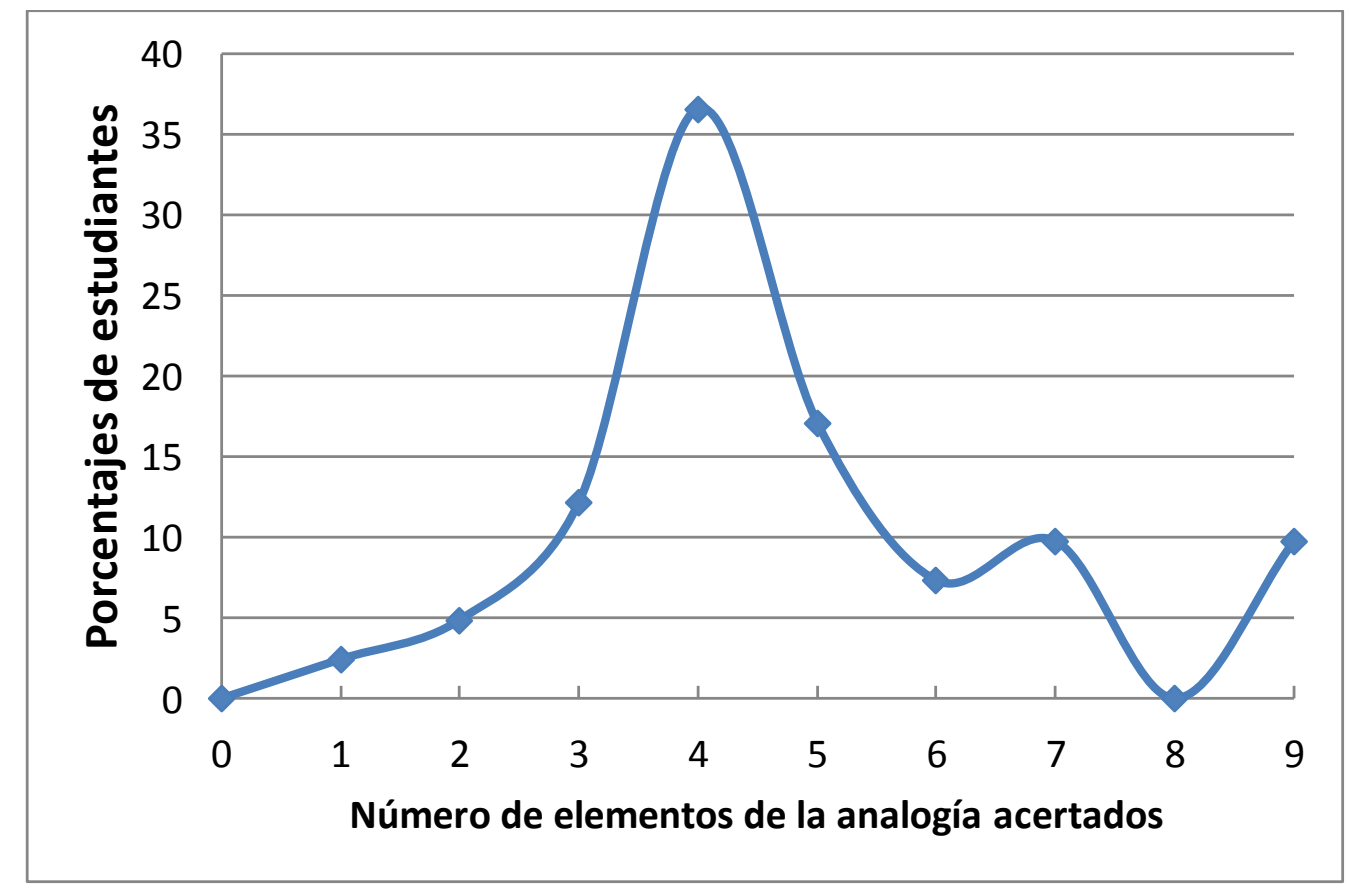

Figura 9: Resultados de la tarea de evaluación de la analogía. Fuente: Datos de la investigación.

La figura 10 realiza un análisis por elementos, mostrando los porcentajes de estudiantes que son capaces de relacionar de forma adecuada cada uno de los elementos de la analogía. 


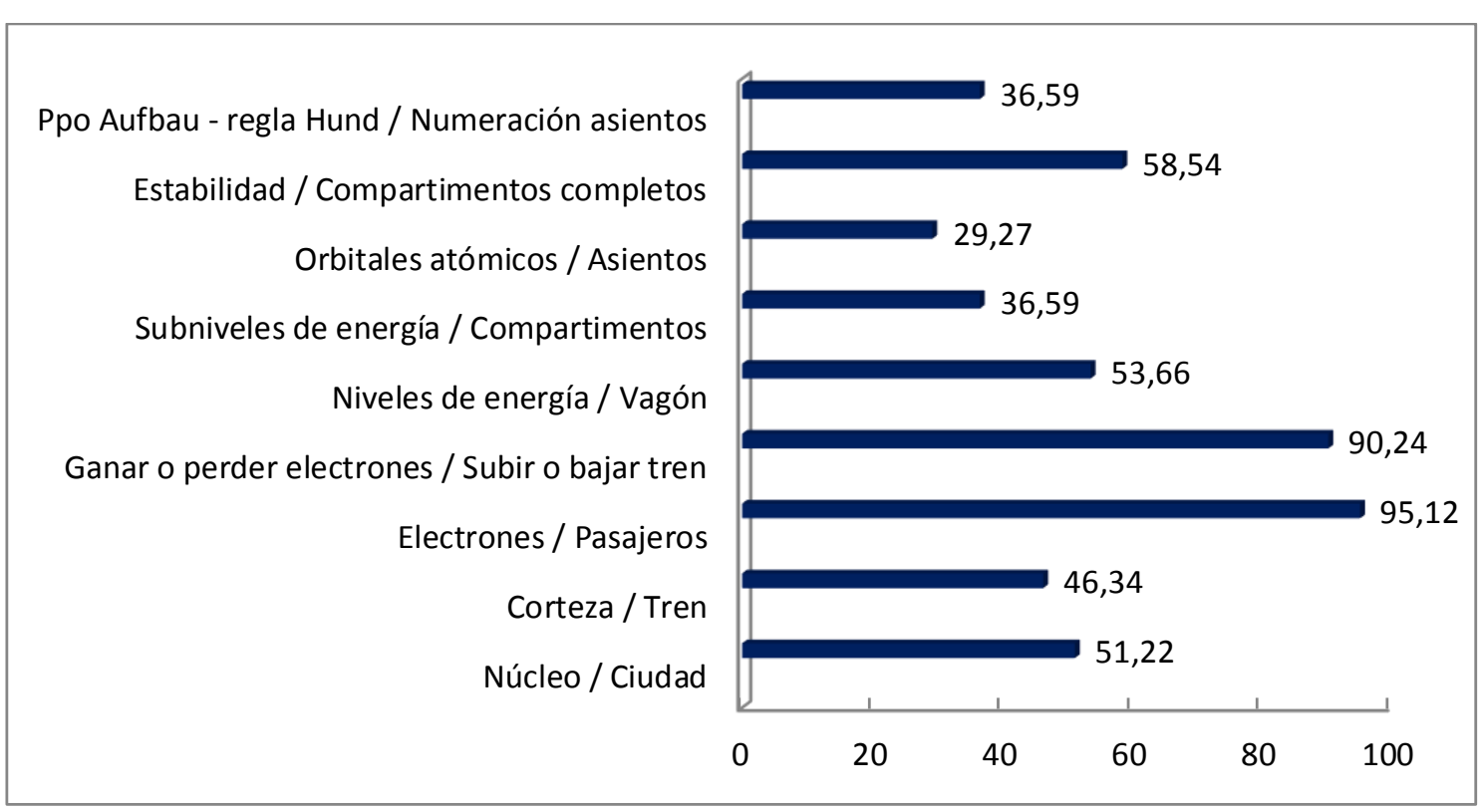

Figura 1o: Porcentajes de estudiantes que indican adecuadamente cada elemento de la analogia. Fuente: Datos de la investigación.

Como se puede observar, no todos los elementos de la analogía son entendidos de igual forma. Así, las analogías usadas para los electrones (95.1\%) o ganar / perder electrones (90.2 \%) son las mejor entendidas, mientras que el concepto de orbital atómico (29.3\%) resulta el más difícil de vincular entre el mundo macroscópico y submicroscópico.

Si consideramos que un elemento de la analogía se ha comprendido adecuadamente si el porcentaje de estudiantes que lo relaciona es superior al 50\%, se puede comprobar que sólo cinco elementos cumplen esta condición (electrones, ganar/perder electrones, estabilidad, niveles de energía y núcleo). Estos resultados ponen de manifiesto la dificultad encontrada también en otros estudios (Pozo y Gómez-Crespo, 1998) que indican que el aprendizaje de estos conceptos requiere de una capacidad de abstracción que no suele estar suficientemente desarrollada en esta etapa educativa.

\section{Evaluación del juego}

No obstante, las percepciones del alumnado sobre el juego son más esperanzadoras, al ser puntuado con una calificación de 8.52 en una escala de o a 10 puntos. Asimismo, los estudiantes valoraron como virtudes clave del juego su interés (100\% de estudiantes lo consideraron interesante o muy interesante), su utilidad $(97,5 \%)$, su sencillez $(95,1 \%)$ y su atractivo (95,1\%). De forma más específica, el 75,6\% de los estudiantes lo consideró muy útil y el $65,8 \%$ muy interesante.

Respecto al aprendizaje, la mayoría del alumnado indicaba acertadamente los conocimientos que había aprendido. Algunas respuestas fueron: "He aprendido la configuración electrónica", "los niveles de organización de los electrones", "el orden de los orbitales", "cómo los niveles se pueden llenar y cómo un átomo puede perder o ganar electrones", etc. Los estudiantes también coincidían en que el aprendizaje a través de juegos acerca la química de una manera más motivadora y amena, expresándolo de esta forma:

"Es una forma más sencilla de aprender química"

"Se puede aprender con otra dinámica en clase y de manera divertida" 
"Con un simple juego, granitos de arroz, un dado y un trozo de cartón he aprendido algo que creía que no iba a entender"

Asimismo, valoraban el recurso como actividad de síntesis de lo aprendido ("Me ha servido para repasar"), que favorece el trabajo colaborativo ("Lo mejor ha sido aprender en grupo con tus compañeros") y ayuda a llevar a la práctica conceptos abstractos ("Se aprende mejor poniéndolo en práctica").

Como propuestas de mejora indicaron que "los granitos de arroz se movían con facilidad", que "las preguntas son siempre las mismas" y el tablero de mesa debía ser un poco más grande.

\section{CONSIDERACIONES FINALES}

Los resultados obtenidos en esta investigación parecen revelar que el uso del juego educativo "El tren orbital" basado en una analogía resulta útil en el aprendizaje de la configuración electrónica, así como para establecer un clima adecuado y motivador para el aprendizaje de la teoría atómica moderna en el aula de secundaria.

No obstante, los resultados de aprendizaje de este estudio piloto también ponen de manifiesto algunas fortalezas y debilidades de la analogía empleada, ya que no todos los elementos utilizados en la analogía son interpretados de igual forma por el alumnado. Así, entre los elementos mejor asimilados se encuentran el concepto de electrón y su ganancia o pérdida por el átomo. Por el contrario, los conceptos relativos a orbitales atómicos, subniveles de energía o principio de Aufbau y regla de Hund son los que muestran más dificultades al extrapolarlos del mundo macroscópico al submicroscópico.

A partir de estos resultados se puede concluir que la analogía ha permitido en la mayoría de los estudiantes un avance parcial en la superación de las dificultades de aprendizaje asociadas a la naturaleza abstracta de los términos que se manejan en este tema, al vincularlos con otros elementos de la vida cotidiana.

Como línea futura de la investigación, se pretenden realizar diferentes mejoras en la analogía y en el juego, así como desarrollar otro estudio con una muestra más amplia de estudiantes que permita generalizar estos resultados.

\section{REFERENCIAS}

ANTUNES, M.; PACHECO, M. A. R.; GIOVANELA, M. Design and Implementation of an Educational Game for Teaching Chemistry in Higher Education. Journal of Chemical Education, V. 89, n. 4, p. 517521, 2012.

CERVELLATI, R. ; PERUGINI, D. The understanding of the atomic orbital concept by Italian high school students. Journal of Chemical Education, V. 58, n. 7, p. 568-569, 1981.

DAGHER, Z. R. Review of studies on the effectiveness of instructional analogies in science education. Science Education, V. 79, n. 3, p. 295-312, 1995.

EISBERG, R.; RESNICK, R. Física Cuántica. México: Limusa, 1994.

FRANCO-MARISCAL, A. J.; OLIVA, J. M.; BLANCO, A.; ESPAÑA, E. A Game-Based Approach To Learning the Idea of Chemical Elements and Their Periodic Classification. Journal of Chemical Education, V. 93, n. 7, p. 1173-119o, 2016.

GARCÍA-CARMONA, A. La estructura electronica de los átomos en la escuela secundaria: un estudio de los niveles de comprensión. Educación Química, V. 17, n. 4, p. 414-423, 2006.

HANSON, R. M. Orbital. Journal of Chemical Education, V. 80, n. 1, p. 109, 2003. 
LEE, O.; EICHINGER, D. C.; ANDERSON, C. W.; BERKHEIMER, G. D.; BLAKESLEE, T. D. Changing middle school students' conceptions of matter and molecules, Journal of Research in Science Teaching, V. 30, n. 3, p. 249-270, 1993.

MORWIEK, J. J. Should orbitals be taught in high school? Journal of Chemical Education, V. 56, n. 4, p. 262-263, 1979.

OLIVA, J. M. Comparando la tabla periódica con un calendario: posibles aportaciones de los estudiantes al diálogo de construcción de analogías en el aula. Educació Química EduQ, V. 6, p. 13-22, 2010.

POZO, J. I.; GÓMEZ-CRESPO, M.A. Aprender y enseñar Ciencia. Madrid (España): Morata, 1998.

ROSADO, L.; GARCÍA-CARMONA, A. El entorno del alumno como marco de referencia en la enseñanza de la Física. En Rosado, L. y cols. (eds.), Didáctica de la Física y sus Nuevas Tendencias. Madrid (España): UNED, pp. 259-312, 2004.

SAMOSHIN, V. V. Orbital models made of plastic soda bottles. Journal of Chemical Education, V. 75, n. 8, p. 985, 1998.

TRINDADE, J.; FIOLHAIS, C. Students' visualization and conceptual understanding of atomic orbitals using a virtual environment. Advanced Learning Technologies, Proceedings. The 3 rd IEEE International Conference on 9-11 July, pp. 298-299, 2003.

Antonio Joaquín Franco-Mariscal: Licenciado en Ciencias Químicas por la Universidad de Cádiz (España) en 1998. Doctor por la Universidad de Cádiz (España) desde 2011. Profesor de Física y Química de Enseñanza Secundaria desde 2001 y Profesor Asociado en el Departamento de Didáctica de las Ciencias Experimentales de la Universidad de Málaga (España) desde 2014.

E-mail: anjoa@uma.es

Rosario Franco-Mariscal: Licenciada en Química por la Universidad de Cádiz (España) en 2000. Doctora por la Universidad de Cádiz desde 2016. Profesora de Física y Química de Enseñanza Secundaria desde 2004 .

E-mail: rosario.franco@uca.es

Guillermo Salas-García. Licenciado en Ciencias Químicas por la Universidad de Cádiz (España) en 1981. Profesor de Física y Química de Enseñanza Secundaria desde 1987.

E-mail: guillermo.salas@iessanctipetri.es 